\title{
On PAPR for Combined Modulation and Access Techniques in Configurable Radio
}

\author{
Eduard Bertran, Oriol Porta, Gabriel Montoro, J.A Delgado-Penin. \\ Department of Signal Theory and Communications \\ Universitat Politècnica de Catalunya (UPC) \\ C/ Esteve Terrades 7, 08860 - Castelldefels - Barcelona, Spain
}

\begin{abstract}
This paper assesses the PAPR for multimode transmitters (WiMAX, WiFi and Bluetooth), considering different combinations of modulations and access techniques (spreading codes and OFDM). Simulations include preamble, header and data fields. Results give hints for tailoring the back-off of power amplifiers, as well as for foreseeing the necessary slew-rate in polar transmitters.
\end{abstract}

Index Terms- Configurable Radio, Multimode, Access Techniques, PAPR, Power Amplifiers.

\section{INTRODUCTION}

Some new communication equipments entail the concurrent operation of different standards, usually in cellular, WAN and WPAN networks, having each application different power, datarate and ranging. Depending on the channel quality, the modulation format is continuously adapted, usually from BPSK to 64-QAM. Besides, the supporting access technology is also diverse, being DSSS (Direct Sequence Spread Spectrum) and OFDM (Orthogonal Frequency Division Multiplexing) the most usual, although FHSS (Frequency Hopping Spread Spectrum) and UWB (Ultra-wideband) are common in the PHY layer of some WPAN's. In addition, operational BW is also scalable in new cellular and WAN standards (i.e., from 1.4 to $20 \mathrm{MHz}$ in $3 \mathrm{GPP}$ standard LTE). Besides, other emerging technologies, such as Cognitive Radio (Dynamic Spectrum Access), also needs to be dynamically reconfigured, thus requiring transmitter front-ends capable to cope with different operating frequencies, powers and bandwidths, as well as modulation formats.

These facts open new challenges in the design of the multimode \& multiband transmitters. Both CDMA-like and multicarrier modulated signals have large peak powers (and consequently large PAPR, peak-to-average power ratio), thus bringing disadvantages for the $\mathrm{A} / \mathrm{D}$ and $\mathrm{D} / \mathrm{A}$ converters, and for the RF power amplifier (PA). With regard to the PA, its biasing is critical to avoid an inefficient use: on one hand, it is the risk of signal saturation, leading to non-linear distortion (ACPR and BER degradation). On the other hand, to bias the PA at large back-off values should reduce the abovementioned risks, but at the price of increasing the power consumption (reduction of power efficiency and battery life). In order to reduce (or avoid) the use of inefficient linear power amplifiers (i.e, class A or B), and taking into account the unsuitability of more efficient switched mode power amplifiers (as class E or F) to cope with non-constant amplitude modulations (except when used within special linearizer structures), the linearity-efficiency tradeoff is being faced in several manners: a) Peak power reduction techniques (signal clipping), based on methods of signal constellation extension or predistortion (i.e, coding, selective mapping, partial transmit sequences or tone reservation [1]). These techniques, software based, kept the BER at the price of sacrificing data throughput. b) The use of PA linearizers, which increase both hardware cost and size, but keeping the data throughput. Among the different structures of PA linearizers, the continuously increasing operational $\mathrm{BW}$, as well as the fast development of digital devices, has practically buried some options and revitalized others such as the Digital Predistorter (DPD) and the Polar Transmitter (PT) [2]. c) By using new modulations, such as Alt-BOC (as in Galileo transmitters) or SC-FDMA (SingleCarrier Frequency Division Multiple Access), used in the uplink of LTE transmitters and showing around $2.4 \mathrm{~dB}$ of PAPR reduction [3].

Whatever the kind of employed PA and the 
way to solve the linearity-efficiency trade-off, PAPR values are crucial. In linear PA structures, because they determine the PA back-off (compromise between signal distortion and power consumption). In signal clipping, because the most accused the PAPR is, the most drastic is the clipping (i.e, the shrinking of the data throughput). Regarding PA linearizers, the effects may be diverse: for example, in DPD the memory effects may be magnified (complicating the predistortion models), while in PT the necessary slew-rate to follow high PAPR in the envelope path may add an additional constrain to the classical BW one.

PAs for multimode\&multiband transmitters may be manufactured in different ways depending on the number of operating bands. If the standards have to operate at different bands, and power efficiency is relevant, one of the structures which allow simultaneous transmissions is the based on a unique broadband power amplifier splitting the different applications with a bank of matched band-pass filters at PA output. Because the input signal of the power amplifier (single or together with a linearizer) is the amount of all the modulated signals from the different active standards to be simultaneously transmitted, PAPR depends on the combination of them. Besides, the total power depends, in real scenarios, of the random phase (coherence) of the signals, so this is a topic suitable to be studied by simulation.

\section{PRELIMINARY WORKS}

Some published works have already assessed the PAPR for different communication standards. In [4] it is evaluated the joint operation of Wi-Fi and WiMAX, or WCDMA and GSM1800 signals, in multimode SDR receivers and transmitters. In [5] a dual-band $802.11 \mathrm{a} / 802.11 \mathrm{~g}$ radio with nine modulation modes is studied, with little information regarding the PAPR: it is stated an approximate value of $2.5 \mathrm{~dB}$ for $802.11 \mathrm{~b}$ in CCK mode at $2.45 \mathrm{GHz}$, and $13 \mathrm{~dB}$ for IEEE 802.11a or 802.11g using the 64-QAM OFDM modulation. This last value for OFDM is very scattered in the literature. For example, in [6] it is stated a load modulation PA for OFDM 802.11g to operate at least $7 \mathrm{~dB}$ backed-off from the maximum rated power. Actually, this value depends on the actual value of the CCDF where it is measured (i.e, $10 \mathrm{~dB}$ at $0.01 \%$ probability on $\mathrm{CCDF}$ is a common figure). Other works have shown the dependence of the PAPR value on preambles transmitted during the same symbol [7].

This paper assess PAPR for different combinations of the standards IEEE $802.11 \mathrm{~b}$ (as an example of WAN that uses two different types of spreading sequences: Barker codes and CCK, both DSSS but with different theoretical PAPR characteristics), IEEE 802.16-2004 (OFDM) -as an example of MAN, and using OFDM-, and Bluetooth (BT, based on standard IEEE 802.15.1), which is an example of WPAN using Frequency Hopping (FHSS) from a previous GFSK modulation. These three applications likely may work together within the same equipment (PDA, laptops...), because of their different goals. The results will present the PAPR of different simultaneous modulations, combined with different media access (DSSS, FHSS and OFDMA). Besides, simulation will consider all the transmitted fields (preambles, headers and data payload), not only the ones supported by efficient coding sequences (data).

\section{SIMULATION MODEL}

The simulation model is based on MatlabSimulink, using envelope models for timeefficiency in simulation runs (each run has been 160128 samples length). To the some purpose, in this first approach, the frequency bands of the three applications are assumed to be the same (i.e, 2,4 GHz for WiFi and BT, also used for some pre-WiMAX operators, as in Russia or Korea). Although the standards using the same band, they may be separated at the received because two of them are spread-spectrum based (SS-FH for BT, and SS-DS for 802-11b), thus allowing bandwidth sharing with WiMAX.

Starting from commercial models provided by Mathworks, they have been adapted (simulation parameters adjustment in order to merge all the models), fitted to IEEE standards and put together in an unique simulation model which include IEEE.11b (WiFi), IEEE 802.16-2004 (OFDM, WiMAX) and BT (considering the three kind of voice-packets, HV1, HV2 and HV3, with different degrees of data protection and consumed power). To previously test the reliability of the model, each of the standards has been simulated deactivating the rest, and the results have been successfully compared with results from other published works [8] and from isolated laboratory measurements (CCDF of $802.11 \mathrm{~b}$ and 802.16). 


\section{RESULTS}

In Fig. 1 it is show the simulated PAPR of the separated operation of WiFi (DSSS), WiMAX (OFDM) and BT (SS-FH) systems, considering all the possible modulations, coding and access techniques. Notice the PAPR is maintained in WiFi when 5.5 Mbps CCK codes (for DQPSK modulation) are used instead of Barker ones (1 and 2 Mbps). In DQPSK+ QPSK+CCK modulation the benefits of using CCK codes are only scarcely perceived as PAPR reduction, while the bit-rate is significantly increased. The result of the comparison within Barker and CCK modulations is coherent with the fact that the CCK codewords are Golay complementary sequences [9] which theoretically have reduced PAPR.

The PAPR of WiMAX signals (with256 subcarriers) results practically independent of the previous data modulation format (Fig. 2), so being only sensitive on the number of used subcarriers. Even the inclusion of preambles produces a PAPR increase of $2-4 \mathrm{~dB}$, a value higher that the effect of the varying modulation formats. Comparing this figure with Fig. 1, it can be seen that only at high PAPR values are perceptible (very slightly) the effects of the modulation formats (previous to the OFDM block).

Finally, Fig.3 and Fig.4, show a twodimensional histogram of the PAPR for different combinations of the modulations within WiMAX and BT (Fig. 3), and WiMAX and WiFi (Fig. 4).

\section{CONCLUSION}

From Fig. 3 it can be stated that in multimode transmitters including BT, the effects of the BT are negligible when this standard is being transmitted not alone. So it is not relevant in the selection of the PA back-off value (obvious regarding the relative small power of BT).

The PAPR values of the different WiFi operation modes supported for both Barker and CCK codes are quite similar (Fig.1) when this standard is used alone. However when it is transmitted together with WiMAX signals, the WiFi modes employing Barker sequences to spread the signal show scarcely worst (higher) PAPR values. And a paradigm happens for WiFi signals which has been spread by means of 11 Mbps CCK codes: while this is the worst situation for the isolated $\mathrm{WiFi}$ operation, it provides the best PAPR values (Fig. 4) when it works together with WiMAX signals in 64 QAM modulation format.

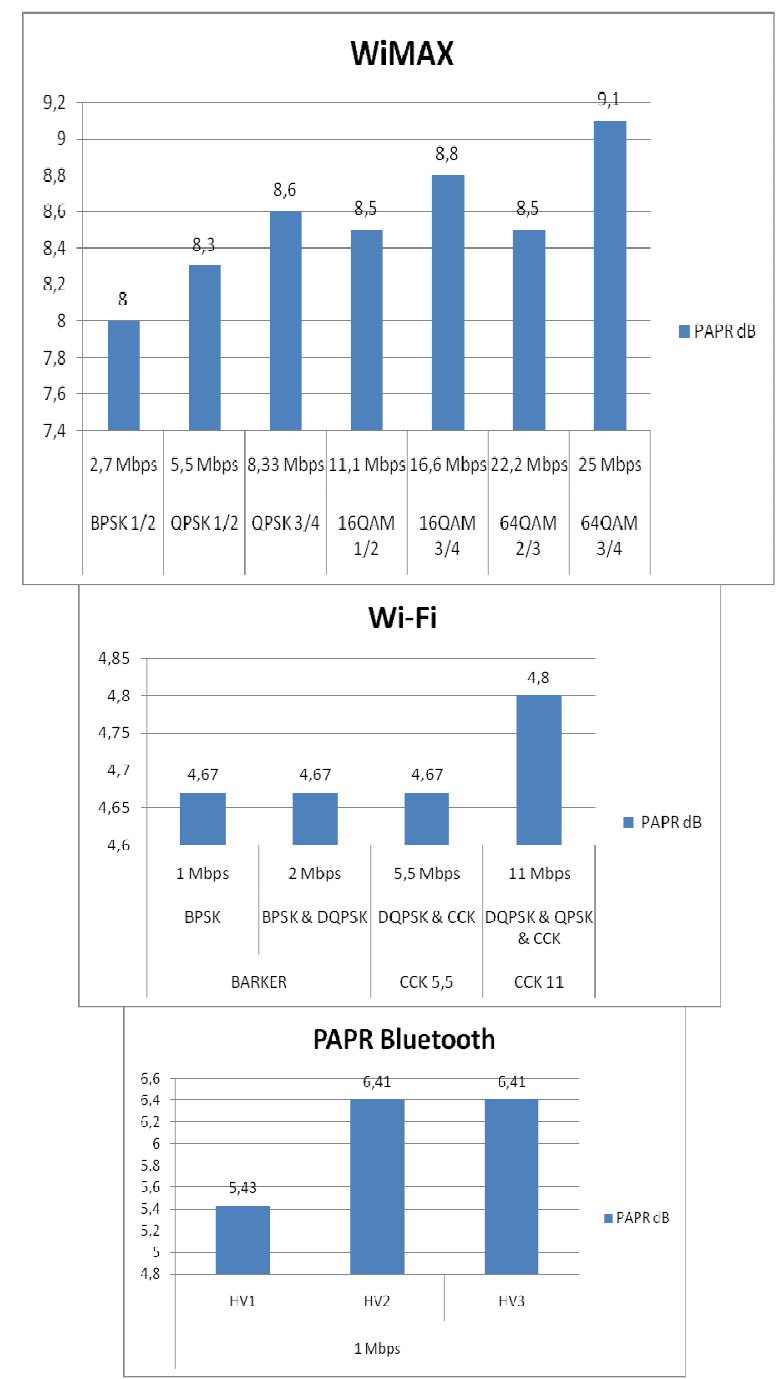

Fig. 1. PAPR for the single standards (including all possible modulations and coding).

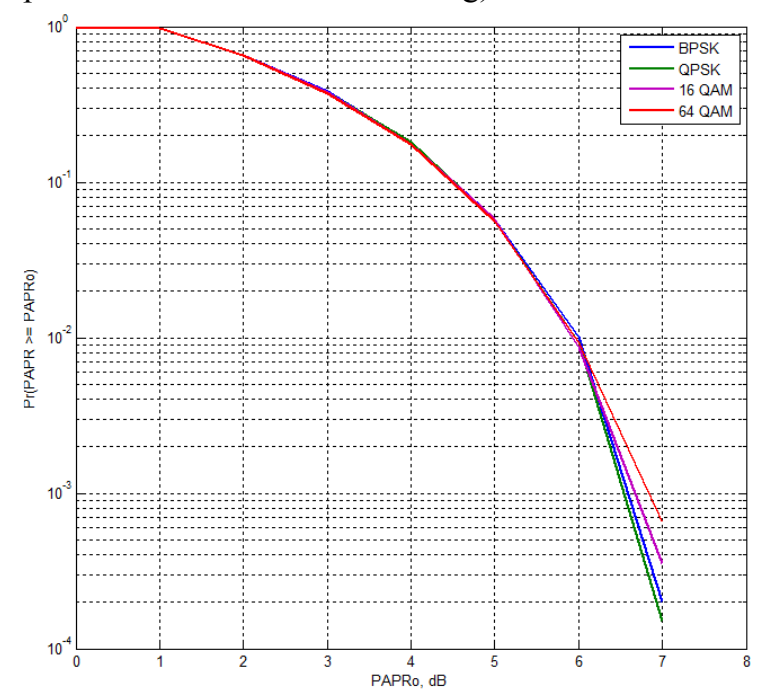

Fig. 2. CCDF of a $\mathrm{N}=256$ WiMAX signal, for different data modulations (preamble + data) 
The aforementioned conclusions are spotted at the qualitative level because they have little effect when they are numerically translated to $\mathrm{dB}$. So, we emphasize that the unique aspect enough significant to reduce PAPR in the multimode transmitter is the number of subcarriers used in the WiMAX subsystem (this number is variable in IEEE 802.16 standard). Hence, it could be suggested to make adaptive amplifiers or linearizers (back-off or slew-rates) only considering this parameter. In $802.11 \mathrm{~b}$, the PAPR it is practically independent of the transmit mode, while in BT the PARP for HV1 mode is around $1 \mathrm{~dB}$ lower.

\section{ACKNOWLEDGMENT}

This work was supported by Spanish Government (MICINN) and FEDER under project TEC2008-06684-C03-03.

\section{REFERENCES}

[1] L. Wang, C. Tellambura, "An Overview of Peak-to Average Power Ratio Reduction Techniques for OFDM systems", IEEE Int. Symp. on Signal Processing and Information Tech., Vancouver, August 2006.

[2] J.Groe, "Polar Transmitters for Wireless Communications", IEEE Communications Magazine, Volume 45n.9, September 2007.

[3] G.Berardinelli, L.A.Maestro, S. Frattasi, M.I. Mahman, P. Mogensen, " OFDMA VS. SC-FDMA: Performance Comparison in Local Area IMT-A Scenarios", IEEE Wireless Communications, October 2008

[4] P. Cruz, N.B.Carvalho, "PAPR Evaluation in MultiMode SDR Transceivers", 38th European Microwave Conference, October 2008, Amsterdam.

[5] R. L. Abrahams, "Troubleshooting Dual-Band WLAN Radios", March 2003, Penton MEdia.

[6] J. Nam, J. Shin and B. Kim, "Load modulation power amplifier with lumped-element combiner for IEEE $802.11 \mathrm{~b} / \mathrm{g}$ WLAN applications" ,Electronics Letters, January 2006, Vol. 42, No. 1.

[7] C. Huo, et alt., "Correction of RS Preamble Configuration Request", IEEE 802.16 Broadband Wireless Access Working Group, Doc: C802.16j-07/318r1, 2007.

[8] S.Coffey, A.Batra, S.Gummadi, M.Shoemake, R. Provencio, C.Heegard, "Comparison of IEEE $802.11 \mathrm{~g}$ Proposals: PBCC, OFDM \& MBCK", DOC: IEEE 802.11-01/064r1, http://mentor.iee.org

[9] W. Jeong, H. Park, H. Lee, S. Hwang, "Performance Improvement Techniques for CCK -OFDM WLAN Modem", IEEE Transactions on Consumer Electronics, Vol. 49, No. 3, August 2003.

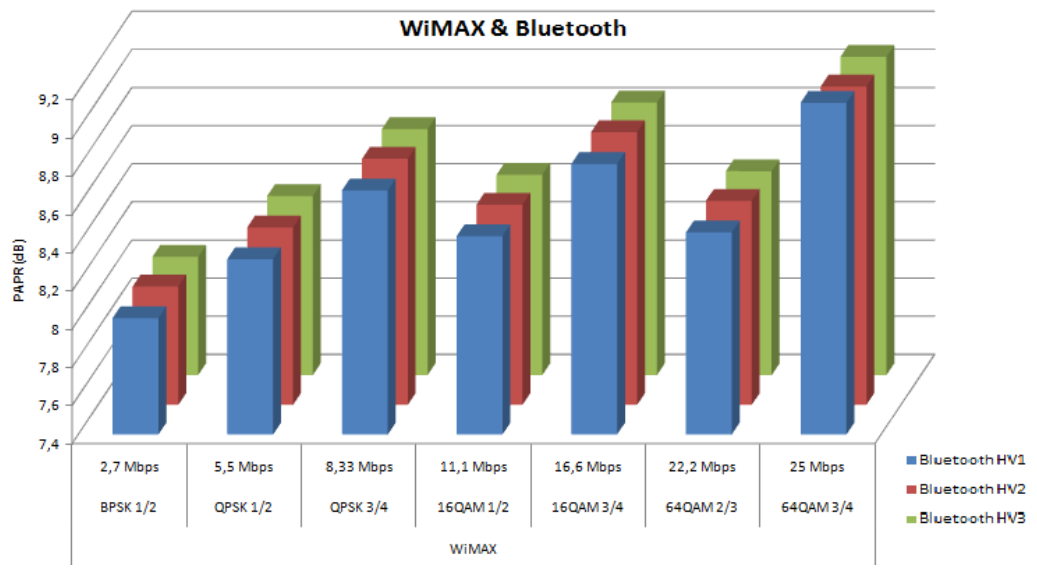

Fig. 3. PAPR for combined WiMAX and Bluetooth transmissions

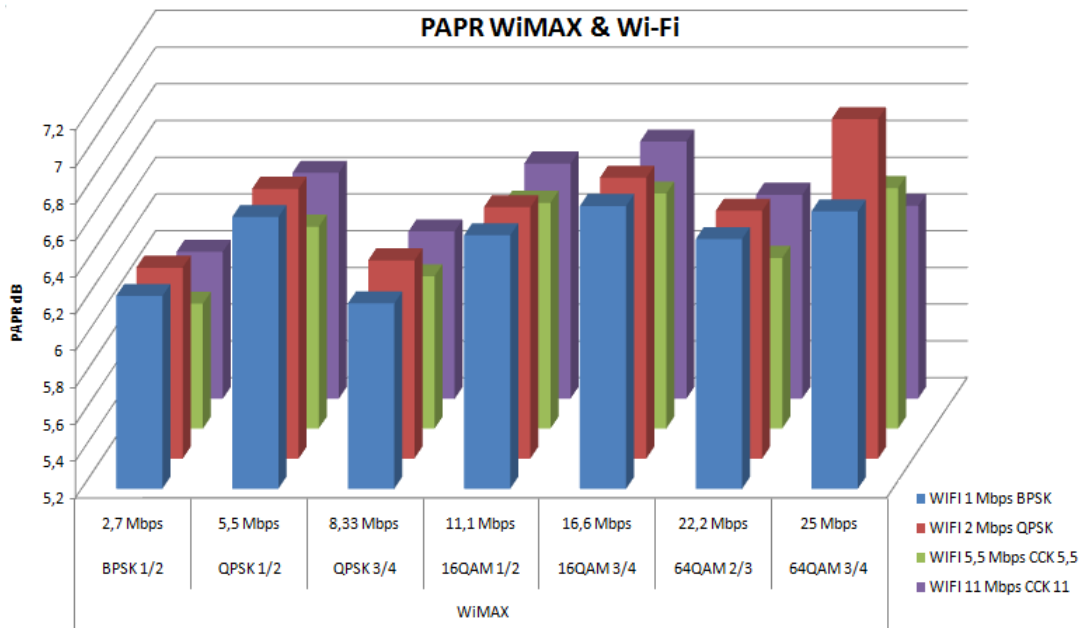

Fig. 4. PAPR for combined WiMAX and WiFi transmissions 